\title{
ОСОБЕННОСТИ СТРУКТУРЫ СЕРДЕЧНОГО РИТМА У ЛИЦ МОЛОДОГО ВОЗРАСТА В ЗАВИСИМОСТИ ОТ ДОМИНИРУЮЩЕГО ТИПА ВЕГЕТАТИВНОЙ НЕРВНОЙ СИСТЕМЫ
}

\author{
(C) Спицин А.П.
}

\author{
Кафедра патофизиологии Кировского государственного медицинского университета, Киров \\ E-mail: kf23@kirovgma.ru
}

\begin{abstract}
Цель работы - определение соотношения основных показателей сердечного ритма у практически здоровых студентов в зависимости от доминирующего типа вегетативной нервной системы (ВНС). У 58 студенток младших курсов медицинского университета оценивали вариабельность ритма сердца по стандартизированной методике, принятой Европейской ассоциацией кардиологии и Североамериканской ассоциацией ритмологии и электрофизиологии. Установлены различия в корреляционных связях показателей центральной гемодинамики с показателями сердечного ритма в зависимости от исходного вегетативного тонуса. Показано, что структура сердечного ритма зависит от доминирующего типа ВНС. Сделано заключение, что отдельные показатели вариабельности сердечного ритма дают представление об активности различных звеньев регуляторных механизмов, а их комплексная оценка позволяет оценить функциональное состоянии организма студентов.
\end{abstract}

Ключевые слова: вариабельность сердечного ритма, центральная гемодинамики, студенты.

\section{PECULIARITIES OF HEART RATE STRUCTURE IN YOUNG PERSONS DEPENDING ON THE DOMINANT TYPE OF THE VERGETATIVE NERVOUS SYSTEM Spitsin A.P.}

Department of Pathological Physiology of Kirov Sate Medical University, Kirov

The purpose of the current research work was to determine correlation of the main indicators of heart rates of practically healthy students depending on the dominant type of the vegetative nervous system. 58 junior medical students of Kirov State Medical University were analyzed. Variability of heart rates was evaluated and estimated according to the European Association of Cardiology and Northern American Association of Rythmology and Electrophysiology. Differences in correlations of central hemodynamics and indicators of heart rates depending on primary vegetative tonus were revealed and determined. It was shown that the structure of heart rates depends on the type of the vegetative nervous system. The conclusion was that some indicators of heart rate variability give presentation on activity of different links of regulatory mechanisms. Their complex evaluation allows to evaluate and estimate functional conditions of students' organisms.

Keywords: heart rate variability, central hemodynamics, students.

Интенсификация учебной деятельности в высших образовательных учреждениях, переход от традиционной организации учебного процесса к инновационным технологиям повышают требования к уровню работоспособности, к состоянию как психического, так и физического здоровья студентов [1].

В связи с этим в последнее десятилетие увеличилась значимость методов для мониторинга и экспресс-оценки состояния здоровья студенческой молодежи [9].

Система кровообращения отличается высокой реактивностью и играет первостепенную роль в адаптационных перестройках функционального состояния организма $[2,8]$.

Одним из наиболее адекватных показателей в оценке функционального состояния организма является сердечный ритм $[4,11,13]$, отражающий фундаментальные соотношения в функционировании не только сердечно-сосудистой системы, но и всего организма в целом, чутко реагирующий на любые стрессорные воздействия [12]. Изменение показателей ВСР сопряжено со многими факторами, действующими на организм, в том числе и с влиянием учебной нагрузки [6].

Анализ вариабельности сердечного ритма (ВСР) позволяет судить о возможности количественной оценки вегетативного гомеостаза по математико-статистическим показателям сердечного ритма, что дает возможность характеризовать изменения уровня функционального состояния организма при отсутствии сдвигов основных физиологических показателей. Гомеостаз может быть сохранен благодаря активизации энергетических механизмов, повышению тонуса симпатической нервной системы $[3,18,19]$. Такого рода изменения нередко возникают в процессе повседневной деятельности студентов. Применение данного подхода особенно важно в случаях длительных психоэмоциональных напряжений, связанных с учебной деятельностью. На сегодняшний день остаются малоизученными особенности вариабельности сердечного ритма в зависимости от доминирующего типа вегетативной регуляции, а также оценка взаимосвязей между АД и показателями сердечного ритма. Очевидно, что исследо- 
вание взаимосвязей АД и ВРС может способствовать пониманию механизмов развития артериальной гипертензии.

Цель работы - определение соотношения основных показателей сердечного ритма у практически здоровых студентов в зависимости от доминирующего типа ВНС, а также взаимосвязей между АД и ВСР.

\section{МАТЕРИАЛЫ И МЕТОДЫ ИССЛЕДОВАНИЯ}

В исследование было включено 58 студенток младших курсов медицинского университета. Средний возраст составил 23,1 $\pm 0,25$ года. Измерение давления проводилось осциллометрическим методом с помощью автоматического тонометра фирмы OMRON с соблюдением протокола ESH/ESC. Критерии установления границ. Все испытуемые предварительно были ознакомлены с содержанием исследования, получено информированное согласие на него. Инструментальные методы исследования включали электрокардиографию. Исследование вариабельности ритма сердца проводили в соответствии с «Рекомендациями рабочей группы Европейского Кардиологического общества и Североамериканского общества стимуляции и электрофизиологии» (1996) на аппарате ВНС Спектр (производство «НейронСофт», г. Иваново) утром через 2 часа после еды и десятиминутного отдыха. Продолжительность фоновой записи электрокардиограммы и при выполнении АОП составляла 5 минут. Анализировались только стационарные участки ритмограмм после устранения всех возможных артефактов и при наличии у пациента синусового ритма. Вариабельность ритма сердца оценивали по стандартизированной методике, принятой Европейской ассоциацией кардиологии и Североамериканской ассоциацией ритмологии и электрофизиологии $[15,16,17]$. При использовании кардиоинтервалографии вычисляли следующие показатели временного анализа: RRNN, мс (среднее значение интервалов RR, Mc), SDNN (CKO), мс (среднее квадратическое отклонение величин NN-интервалов анализируемой записи), RMSSD, мс (корень квадратный из средней суммы квадратов разностей величин соседних пар NN-интервалов), pNN50, \% (процент пар последовательных интервалов $\mathrm{NN}$, которые различаются более чем на 50 мс), показатели спектрального анализа: ТP (Total Power), мс ${ }^{2}$ (общая мощность в диапазоне частот $\leq 0,4$ Гц), HF (High Frequency), мс $^{2}$ (мощность в диапазоне высоких $(0,15-0,4$ Гц) частот (волны длительностью 2,5-6,5 сек), LF (Low Frequency), мс ${ }^{2}$ (мощность в диапазоне низких $(0,04-0,15$ Гц) частот (волны длительностью
6,5-25 сек), VLF (Very Low Frequency), мс ${ }^{2}$ (мощность в диапазоне очень низких $(\leq 0,04$ Гц $)$ частот (волны длительностью более 25 сек), HFnorm, \% (нормализованная мощность в диапазоне высоких частот) - отражает относительный вклад HFкомпонента в общую мощность за вычетом VLFкомпонента, LFnorm, \% (нормализованная мощность в диапазоне низких частот) - отражает относительный вклад LF-компонента в общую мощность за вычетом VLF-компонента; LF/HF; показатели кардиоинтервалографии по Р.М. Баевскому: АМо (амплитуда моды), \% (количество кардиоинтервалов, соответствующих диапазону моды (в \%); ВПР (вегетативный показатель ритма), у.е. (ВПР = 1/Mo $\times \mathrm{BP}) ;$ ИН (индекс напряжения регуляторных систем), усл. ед.; (ИН = $\mathrm{AMo} / 2 \times \mathrm{BP} \times \mathrm{Mo})$. Считается, что отдельные показатели ВСР дают представление об активности различных звеньев регуляторных механизмов, а их комплексная оценка дает представления о функциональном состоянии $[13,15,20]$. При оценке вегетативного тонуса ориентировались на показатели (RRNN, SDNN, ИН), согласно рекомендациям Р.М. Баевского.

Статистическая обработка полученных данных была проведена с использованием компьютерных пакетов Excel и Statistica Advanced 10 for Windows RU, лицензионный номер 136-394-673. Полученные данные представлены в виде средних значений с их ошибкой средней $(\mathrm{M} \pm \mathrm{m})$. При обработке полученных результатов использовали непараметрические методы статистики; для определения различий между группами - критерий Манна-Уитни; для выявления корреляционных зависимостей между различными параметрами коэффициент корреляции Спирмена. Достоверными считали различия и корреляции при $р<0,05$.

\section{РЕЗУЛЬТАТЫ ИССЛЕДОВАНИЯ И ИХ ОБСУЖДЕНИЯ}

Среднее значение RR интервалов в подгруппе симпатотоников $(\mathrm{n}=15)$ составило $688,07 \pm 9,00$ мс. Нормальные значения SDNN (от 40 до 80 мс) зарегистрированы в $13,79 \%$ случаев (8 человек). Нормальные значения rMSSD (от 20 до 50 мс) зарегистрированы в 20,69\% случаев (12 человек), сниженные - в 5,17\% (3 человека). Анализ pNN50 выявил несколько иную картину: нормальные значения pNN50 (больше 15\%) не зарегистрированы, сниженные - в 25,86\% (15 человек). Снижение rMSSD и, особенно, pNN50 свидетельствует о снижении активности парасимпатического звена вегетативной регуляции у студентов с доминированием симпатического отдела ВНС. На усиление симпатических влияний в регуляции сердечного ритма у данного контингента указы- 
вает и увеличение значений АМо и индекса напряжения (ИН). АМо свыше $40 \%$ выявлена в $17,24 \%$ случаев (10 человек), а ИН больше 120 усл. ед. - в 18,96\% случаев (11 человек).

Следующим этапом нами проведен спектральный анализ. Известно, что спектральный анализ позволяет исследовать активность отдельных уровней управления ритмом сердца $[3,13$, 15].

Сравнение показателей VLF, LF, HF у симпатотоников, выраженных в процентах от суммарной мощности спектра, выявило существенное увеличение доли VLF (в среднем 43,04 $\pm 3,81 \%$ ).

Происхождение медленных волн 2 порядка (VLF) в настоящее время представляется наименее изученным. Полагают, что, вероятно, оно отражает активность межсистемного уровня регуляции. Так отмечено увеличение мощности этих волн у пациентов с психовегетативным синдромом, при этом выявлена корреляция с представленностью тревожно-депрессивных расстройств, определяемых по тесту Спилбергера и Бека [14].

На долю НF приходилось всего 19,82 2 2,52\%). Увеличение доли LF $(37,085 \pm 4,54 \%)$ указывает на увеличение контролирующих влияний на вазомоторный центр ствола мозга со стороны вышележащих отделов центральной нервной системы, а также о росте модулирующих влияний симпатического отдела ВНС на сердечный ритм. На усиление симпатических влияний также указывают и различия в значениях LF и $\mathrm{HF}$, выраженные в нормализованных единицах $(62,99 \pm 4,95$ и $37,16 \pm 4,95$ соответственно) и соотношение LF/HF $(3,13 \pm 0,92)$. Увеличение индекса централизации $(6,44 \pm 1,25)$ указывают на переориентацию автономных механизмов регуляции сердечным ритмом к более высоким центральным механизмам регуляции.

У симпатотоников выявлена положительная корреляционная связь АДС только с АДД ( $\mathrm{r}=0,68$; $\mathrm{p}=0,005)$, а с временными и спектральными показателями ВСР достоверных связей не выявлено. У диастолического артериального давления была определенная связь только со средним значением $\mathrm{RR}$-интервалов ( $\mathrm{r}=-0,44 ; \mathrm{p}=0,09)$. Изменение корреляционных взаимосвязей между АД и показателями вариабельности сердечного ритма выявлено у больных с артериальной гипертензией [11].

Среднее значение RR-интервалов было достоверно связано с rMSSD $(\mathrm{r}=0,58 ; \mathrm{p}=0,02)$, АMо $(\mathrm{r}=-0,62 ; \mathrm{p}=0,014), \mathrm{B \Pi P}(\mathrm{r}=-0,65 ; \mathrm{p}=0,008), \mathrm{TP}$ $(\mathrm{r}=0,61 ; \mathrm{p}=0,014), \mathrm{VLF} \quad(\mathrm{r}=0,51 ; \mathrm{p}=0,053), \quad \mathrm{HF}$ $(\mathrm{r}=0,58 ; \mathrm{p}=0,024)$. Из анализа корреляционных связей видно, что в конечном итоге длительность кардиоинтервалов определяется как симпатическим, так и парасимпатическим отделами ВНС.
Определенное значение имеет и центральный контур регуляции.

Проведенный корреляционный анализ между SDNN (общей вариабельностью сердечного ритма) и параметрами, отражающими симпатическую активность (АМо, ВПР, ТP, LF), указывает на тесную корреляционную связь. Причем эта связь оказалась сильнее, чем связи SDNN с параметрами, отражающими парасимпатическую активность (RMSSD, pNN50, TP, HF). Коэффициенты корреляции между SDNN и АМо составили $\mathrm{r}=-0,96(\mathrm{p}=0,0000), \mathrm{SDNN}$ и LF $-\mathrm{r}=0,8(\mathrm{p}=0,0003)$, между SDNN и RMSSD - r=0,71 ( $\mathrm{p}=0,002), \mathrm{SDNN}$ и $\mathrm{pNN} 50-\mathrm{r}=0,76(\mathrm{p}=0,0009), \mathrm{SDNN}$ и ТР $-\mathrm{r}=0,86$ $(p=0,00004), \operatorname{SDNN}$ и HF $-r=0,57(p=0,026)$.

Среднее значение RR интервалов в подгруппе

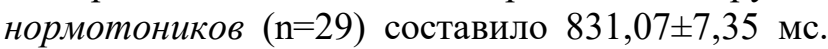
Нормальные значения SDNN (от 40 до 80 мс) зарегистрированы в $36,21 \%$ случаев (21 человек). Нормальные значения rMSSD (от 20 до 50 мс) зарегистрированы в 18,97\% случаев (11 человек), повышенные - в 31,03\% (18 человек). Анализ pNN50 выявил несколько иную картину: нормальные значения pNN50 (больше 15\%) зарегистрированы в 29,3\% случаев (17 человек), сниженные - в 20,69\% (12 человек). Снижение rMSSD и, особенно, pNN50 свидетельствует о снижении активности парасимпатического звена вегетативной регуляции. Увеличение АМо свыше 40\% выявлено у в 17,24\% (10 человек), а ИН больше 120 усл. ед. - в 5,17\% случаев (3 человека).

Сравнение показателей VLF, LF, HF у нормотоников, выраженных в процентах от суммарной мощности спектра, выявило практически равный вклад в регуляцию сердечного ритма. На долю

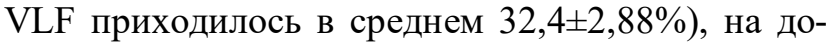
лю $\mathrm{HF}(34,76 \pm 2,33 \%)$ и LF $(32,79 \pm 2,54 \%)$.

У нормотоников, по сравнению с симпатониками, выявлена более достоверная корреляционная связь между АДС и АДД ( $\mathrm{r}=0,66 ; \mathrm{p}=0,00009)$, a с временными и спектральными показателями ВСР достоверных связей не выявлено. Определенная связь АДС выявлена только с LF (r=0,36; $\mathrm{p}=0,056)$. У диастолического артериального давления была слабая отрицательная связь только с HF ( $\mathrm{r}=-0,35 ; \mathrm{p}=0,061)$.

Корреляционный анализ показал, что значение RRNN у нормотоников в наибольшей степени зависит от величины RMSSD ( $\mathrm{r}=0,52 ; \mathrm{p}=0,004$, $\mathrm{pNN} 50(\mathrm{r}=0,51 ; \mathrm{p}=0,0044), \mathrm{TP} \quad(\mathrm{r}=0,42 ; \mathrm{p}=0,023)$, $\mathrm{HF}(\mathrm{r}=0,54 ; \mathrm{p}=0,002)$ и в меньшей степени коррелирует с AMo ( $\mathrm{r}=-0,37 ; \mathrm{p}=0,046), \mathrm{LF} \quad(\mathrm{r}=0,27$; $\mathrm{p}=0,16) \mathrm{VLF} \%(\mathrm{r}=-0,35 ; \mathrm{p}=0,063)$, что отражает более выраженную парасимпатическую активность. 
Среднее значение RR интервалов в подгруппе

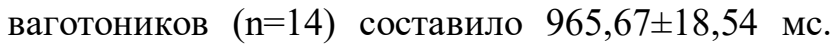
Нормальные значения SDNN (от 40 до 80 мс) зарегистрированы в $15,52 \%$ случаев (9 человек). Нормальные значения rMSSD (от 20 до 50 мс) зарегистрированы в $3,45 \%$ случаев (2 человека), повышенные - в 20,69\% (12 человек). Анализ pNN50 выявил несколько иную картину: нормальные значения pNN50 (больше 15\%) зарегистрированы в $15,53 \%$ случаев (9 человек), сниженные - в $5,17 \%$ (3 человека). Увеличение АМо свыше $40 \%$ выявлено в 7,0\% (4 человека), а ИН больше 120 усл. ед. - не выявлен. На долю VLF приходилось в среднем $27,88 \pm 3,71$ ), на долю НF $40,43-3,83 \%$ и LF - 31,68 $44,2 \%$. На изменение временных и спектральных показателей в структуре сердечного ритма у студентов в течение учебного года, а также во время экзаменов указывают многие авторы [7].

У ваготоников, по сравнению с симпатотониками и нормотониками не выявлено корреляционной связи между АДС и АДД.

Определенная связь АДС выявлена с ЧСС $(\mathrm{r}=-0,49 ; \mathrm{p}=0,07)$ и достоверная связь с модой $(\mathrm{r}=0,54 ; \mathrm{p}=0,046)$, а с спектральными показателями ВСР достоверных связей не выявлено). У диастолического артериального давления была достоверная связь только с индексом напряжения $(\mathrm{r}=0,54 ; \mathrm{p}=0,047)$ и определенная связь с $\mathrm{MMSD}$ $(\mathrm{r}=-0,48 ; \mathrm{p}=0,086)$ и вариационным размахом $(\mathrm{r}=-0,52 ; \mathrm{p}=0,055)$, а с спектральными показателями ВСР достоверных связей не выявлено.

Проведенный корреляционный анализ между SDNN (общей вариабельностью сердечного ритма) и параметрами, отражающими парасимпатическую активность (RMSSD, pNN50, TP, HF), показал следующее. Выявлены достоверные связи SDNN c RMSSD $(\mathrm{r}=0,95 ; \mathrm{p}=0,0000)$. Связь c pNN50 оказалась слабее, однако была достоверной $(\mathrm{r}=0,59 ; \mathrm{p}=0,026)$. Корреляционные связи SDNN с ВПР ( $\mathrm{r}=-0,80 ; \mathrm{p}=0,0005)$ и ИН $(\mathrm{r}=-0,87$; $\mathrm{p}=0,000047)$ оказались достаточно сильными. Более сильными оказались связи SDNN с VLF и LF $(\mathrm{r}=0,85 ; \mathrm{p}=0,0001$ и $\mathrm{r}=0,82 ; \mathrm{p}=0,0003$ соответственно). Корреляционная связь SDNN с высокочастотным спектром, напротив, оказалась более слабой $(\mathrm{r}=0,75 ; \mathrm{p}=0,002)$. Полученные данные свидетельствуют о том, что у студентов имеет место вегетативный дисбаланс с преобладанием симпатической активности при отчетливом усилении центральных влияний. Подобную картину изменений вариабельности сердечного ритма, которая указывает на напряжение в регуляции сердечно-сосудистой системы у студентов обоего пола и снижение функциональных резервов их организма в процессе учебы отмечают и другие исследователи $[5,6]$.
Таким образом, в ходе проведенных исследований выявлены индивидуально-типологические различия параметров вариабельности сердечного ритма у лиц молодого возраста (студенты) при разном доминирующем типе вегетативной регуляции. Показаны различия в корреляционных связях параметров ВСР с показателями гемодинамики с различным исходным вегетативным тонусом. Можно предположить, что дальнейшие исследования вариабельности сердечного ритма в сочетании с показателями гемодинамики позволят получить объективные характеристики функционального состояния организма, а также расширят понимание механизмов повышения артериального давления.

На основании полученных данных можно сделать следующие выводы:

1. Для лиц с доминированием симпатического отдела BHC характерно снижение rMSSD и, особенно, pNN50, существенное увеличение доли VLF (в среднем 43,04 $\pm 3,81$ ), увеличение индекса централизации $(6,44 \pm 1,25)$, что указывают на переориентацию автономных механизмов регуляции сердечным ритмом к более высоким центральным механизмам регуляции. Наиболее высокая негативная корреляция была выявлена между АДД и средним значением RR-интервалов $(\mathrm{r}=-0,44 ; \mathrm{p}=0,09)$.

2. У нормотоников выявлен практически равный вклад VLF, LF, HF в регуляцию сердечного ритма. Снижение rMSSD свидетельствует о снижении активности парасимпатического звена вегетативной регуляции. Определенная корреляционная связь АДС выявлена только с LF $(\mathrm{r}=0,36$; $\mathrm{p}=0,056)$. У диастолического артериального давления была слабая отрицательная связь только с $\mathrm{HF}(\mathrm{r}=-0,35 ; \mathrm{p}=0,061)$.

3. У ваготоников, по сравнению с симпатотониками и нормотониками, не выявлено корреляционной связи между АДС и АДД. Определенная отрицательная связь АДС выявлена только с ЧСС $(\mathrm{r}=-0,49 ; \mathrm{p}=0,07)$, а с спектральными показателями ВСР достоверных связей не установлено.

\section{ЛИТЕРАТУРА}

1. Андреев Д.А., Нестеренко А.И., Васильев В.Н. Физиологическая, психологическая и профессиональная адаптация студентов в медицинских учебных заведениях // Физиология человека. - 2007. Т. 33, № 4. - C. 128 .

2. Артеменков A.A. Изменения вегетативных функций у студентов при адаптации к умственным нагрузкам // Гигиена и санитария. - 2007. - № 1. C. $62-64$

3. Баевский Р.М., Черникова А.Г. Оценка адаптационного риска в системе индивидуального донозологического контроля // Российский физиологиче- 
ский журнал им. И.М. Сеченова. - 2014. - Т. 100, № 10. - С. 1180-1194.

4. Гаврилова Е.A. Спорт, стресс, вариабельность: монография. - М. : Спорт, 2015.

5. Гурова О.А., Тарбаева Е.А., Сафронова Е.Ю. Дневная динамика вариабельности сердечного ритма у студентов // Новые исследования. - 2012. № 3(30). - С. 32-36.

6. Димитриев Д.А., Димитриев А.Д., Карпенко Ю.Д., Саперова E.B. Влияние экзаменационного стресса и психоэмоциональных особенностей на уровень артериального давления и регуляцию сердечного ритма у студенток // Физиология человека. 2008. - Т. 34, № 5. - С. 89-96.

7. Кашина Ю.В.Оценка адаптации студентов в начале и в конце учебного года по вариабельности ритма сердца // Фундаментальные исследования. 2014. - № 10, Ч. 3. - С. 514-517.

8. Киселев А.Р, Гриднев В.И., Посненкова О.М. Оценка адаптационного резерва вегетативной регуляции сердца на основе динамических показателей вариабельности сердечного ритма // Кардиоваскулярная терапия и профилактика.- 2006. - Т. 5, № 6, Приложение 1.- С. 179.

9. Косолапов А.Б., Цыбулько Е.И., Юдина Т.П., Макарова E.B. Разработка системы скринингового контроля за состоянием здоровья студентов и методик его коррекции на основе принципов пищевой и физиотерапевтической санации // Валеология. 2002. - № 3. - С. 37-43.

10. Котельников С.А., Ноздрачев А.Д., Одинак М.М., Шустов Е.Б., Коваленко И.Ю., Давыденко В.Ю. Вариабельность ритма сердца: представления о механизмах // Физиология человека. - 2002. T. 28, № 1. - C. 130.

11. Першина Т.А., Спицин А.П. Особенности гемодинамики и вариабельности сердечного ритма у лиц мужского пола с повышенным артериальным давлением с симпатическим типом регуляции в разных возрастных группах // Вятский медицинский вестник. - 2014. - № 1. - С. 4-8.

12. Севрюкова Г.А., Коновалова Г.М. Функциональное состояние и регуляторно-адаптивные возможности организма человека: монография. - Волгоград : ВолгГТУ, 2015. - 104 с.
13. Солонин Ю.Г., Черникова А.Г., Марков А.Л., Варламова Н.Г., Бойко Е.Р. Годовая динамика показателей вариабельности сердечного ритма у участников северной группы проекта «Марс-500» при длительном мониторинге // Авиакосмическая и экологическая медицина. -2013. - № 4 - С. 141-42.

14. Хаспекова Н.Б. Диагностическая информативность мониторирования вариабельности ритма сердца // Вестник аритмологии. - 2003. - № 32. - С. 15-23.

15. Bauer A., Malik M., Schmidt G., Barthel P., Bonnemeier H., Cygankiewicz I., Guzik P., Lombardi F., Müller A., Oto A., Schneider R., Watanabe M., Wichterle $D$., Zareba $W$. Heart rate turbulence: standards of measurement, physiological interpretation, and clinical use: International Society for Holter and Noninvasive Electrophysiology Consensus // J. Am. Coll. Cardiology. - 2008. - Vol. 52, N 17. - P. 1353-1365. doi: 10.1016/j.jacc.2008.07.041.

16. Heart rate variability: standards of measurement, physiological interpretation and clinical use. Task Force of the European Society of Cardiology and the North American Society of Pacing and Electrophysiology // Circulation. - 1996. - Vol. 93, N 5. - P. 1043-1065.

17. Kleiger R.E., Stein P.K., Bigger J.T. Jr. Heart rate variability: measurement and clinical utility // Annals of Noninvasive Electrocardiology. - 2005. - Vol. 10, N 1. - P. 88-101. - doi: 10.1111/j.1542474X.2005.10101.x.

18. Parati G., Mancia G., Di Rienzo M., Castiglioni P., Taylor J.A., Studinger P. Point: cardiovascular variability is/is not an index of autonomic control of circulation // J Appl Physiol. - 2006. - Vol. 101, N 2. - P. 676-682. - doi: 10.1152/japplphysiol.00446.2006.

19. Rickards C.A., Ryan K.L., Convertino V.A. Characterization of common measures of heart period variability in healthy human subjects: implications for patient monitoring // J Clin Monit Comput. - 2010 .Vol. 24, N 1. - P. 61-70. - doi: 10.1007/s10877-0099210-z.

20. Stein K., Yanez D., Domitrovich P.P., Gottdiener J., Chaves P., Kronmal R., Rautaharju P. Heart rate variability is confounded by the presence of erratic sinus rhythm // Computers in Cardiology. - 2002. - Vol. 29, N 4. - P. 669-672. 